\title{
Corneal response to rigid contact lens wear
}

\author{
J. P. G. BERGMANSON ${ }^{1}$ AND L. W-F. CHU ${ }^{2}$ \\ From the ${ }^{1}$ University of Houston College of Optometry, Houston, Texas 77004, and the \\ ${ }^{2}$ Department of Neurobiology and Anatomy, University of Texas, Health Science Center, \\ Medical School at Houston, Texas 77030, USA
}

SUMmARY Three adult rhesus monkeys were subjected to 2 and 24 hours of polymethylmethacrylate (PMMA) contact lens wear. The induced corneal changes were examined with the electron microscope. Mild epithelial oedema as well as early degenerative cell changes was present already after 2 hours' wear. Rigid lens wear for 24 hours produced more severe oedema and cell alterations together with premature cell loss and ultimately, in areas of lens bearing, corneal denuding. Only the monkeys wearing contact lenses for 24 hours had significant stromal swelling, which was primarily evident in the posterior region, while the anterior limiting lamina remained unaffected. The stromal swelling was patchy and mainly around keratocytes and between lamellae, while fluid within the lamellae was evident only occasionally in posterior stroma. Changes among keratocytes were evident, especially posteriorly, where reaction was frequently severe. Endothelial reaction was restricted to a limited fluid uptake in the 24-hour-wear experiment. In addition there was in these monkeys an apparent loosening of the endothelial adhesion to the posterior limiting lamina. It is concluded that the oedematous epithelium undergoes cell shrinkage and flattening, which is compensated for by an uptake of fluid. The uptake of fluid maintains the overall normal thickness of the epithelium. The conclusion is supported by other studies, where the normal thickness of oedematous epithelium has been shown by pachometry. The results in the present study further suggest that stromal oedema in the contact lens wearer is a result of a relative loss of endothelial function, leading to a swelling that moves in a posterior to anterior direction.

Corneal oedema is recognised clinically as a relative loss of transparency and as an increased anteroposterior thickness. Stromal oedema of the rabbit cornea was studied with the electron microscope after injuring the epithelium and/or endothelium with a cryoapplicator. ${ }^{1}$ It was suggested that early oedema is produced by an irregular increase in the the interfibrillar distance, while more long-term stromal swelling also creates collagen free spaces. This pattern of stromal oedema has also been described in pathological human corneas. ${ }^{2}$

In the pathologically swollen epithelium fluid had entered to form spaces between the cells which remained attached to each other through normal appearing desmosomes. ${ }^{23}$ While contact-lensinduced corneal epithelial oedema is clinically observable by the slit-lamp, it appears that anoxia

Correspondence to Dr J. P. G. Bergmanson. produces a negligible increase in thickness of the epithelium. ${ }^{45}$ Contact-lens-induced epithelial trauma is readily visualised through fluorescein staining of the cornea. A histological description of such injuries has recently been provided by Bergmanson and $\mathrm{Chu}^{6}{ }^{6}$

Changes in the posterior cornea of contact lens wearers are known to occur. Ruben ${ }^{7}$ describes vertical folds in the area of posterior limiting lamina (Descemet's membrane) and an alteration in the shape of the endothelial cell. The vertical folds appearing in the posterior cornea in some contact lens wearers have been shown to be secondary to corneal swelling due to oxygen deprivation. ${ }^{8}$

Contact-lens-induced corneal changes have been described with the use of the slit-lamp but not studied in detail by means of histological techniques: The objectives of the present study are to describe the corneal changes provoked by contact lens wear and to establish if these changes are comparable to those 
reported in the pathologically oedematous cornea. Morphological observations may also provide evidence for what occurs in the epithelial oedema that may be seen in the slit-lamp but does not produce a measurable increase in thickness. By varying the duration of wearing the contact lenses it is hoped that early changes may be distinguished from late changes.

\section{Material and methods}

Three young adult rhesus monkeys (Macaca mulatta) were fitted with rigid contact lenses. The PMMA lenses had smooth transitions and well-rounded edges, and good centration was facilitated by fitting the lenses marginally steep. Two of the monkeys wore the lenses continuously for 24 hours while the third wore them for 2 hours. The fourth served as a control.

After removal of the contact lenses the eyes were enucleated under deep anaesthesia (pentobarbitone sodium) and immediately placed in fixative (3\% glutaraldehyde in $0 \cdot 1 \mathrm{M}$ cacodylate buffer at $\mathrm{pH} 7.2$ ), in which the whole corneas were excised. Each cornea was divided into sectors with the apex towards the central cornea. Sectors from different orientations, amounting to approximately a quarter of the whole cornea, were examined in the electron microscope.

After post-staining in $1 \%$ osmium tetroxide for 3 hours the tissues were dehydrated through an alcohol series and embedded in Epon 812. Semithin $(1 \mu \mathrm{m})$ sections obtained from central and peripheral cornea were stained in toluidine blue and evaluated under the light microscope. Thin sections recovered from the same blocks as the light microscopic preparations were stained with $3.5 \%$ uranyl acetate and Reynold's lead citrate. The observations were made with a Jeol $100 \mathrm{C}$ electron microscope.

\section{Results}

\section{E PITH ELIUM}

In the control eyes the corneal epithelium was composed of 5 to 6 layers of tightly packed cells with no interstitial spaces (Fig. 1). The undulating epithelial plasmalemma was closely followed by the neighbouring cell. Numerous desmosomal junctions were observed throughout the epithelium.

After 2 hours of rigid contact lens wear mild epithelial oedema was observed (Fig. 2). Throughout the full thickness of the epithelium spaces filled with a granular material were found within the cells as well as between them. At this stage lipid bodies were also present within the cells and occupied all the epithelial layers. The contact lens wear had also reduced the number of microvilli along the surface.

Eyes that wore contact lenses for 24 hours showed all of the above described changes but they were more pronounced at this point (Figs. 3, 4, 5). Spaces between cells were observed to be greater, with an apparent stretching of the cytoplasm adjacent to desmosomes, resulting in the appearance of fingerlike projections from the cells. Epithelial cells in areas of more severe oedema had a reduced number of desmosomal contacts with neighbouring cells at the same time as microvillus-like protoplasmic extensions appeared (Figs. 3, 4, 5). Cell death had reduced the epithelial thickness by one or more layers of cells in areas of severe oedema (Fig. 6). The remaining cells took up a distorted shape, and many lysosome-like bodies developed (Figs. 5, 6). Although the epithelial basement membrane lost its linear outline, it was never found to be penetrated in any region. The flattened basal cells closely followed the outline of the basement membrane to which they remained attached through the hemidesmosomes (Fig. 6). After 24 hours there were areas of the cornea which were devoid of epithelium (Fig. 7). These were regions where bearing of the contact lens had been most pronounced. In these areas the basal cell would rupture leaving the internal plasmalemma still adherent to the basement membrane. No invading cells were found in the epithelium in either the mildly or severely oedematous epithelium.

\section{STROMA}

In the control eyes the anterior limiting lamina (Bowman's membrane) was formed by fine randomly orientated collagen fibres (Fig. 1). This layer showed no detectable changes in the oedematous cornea (Figs. 2. 6. 7). The eyes which had worn contact lenses for 24 hours showed stromal oedema around the keratocytes, along which large spaces filled with a granular material had been formed (Figs. 9, 10). The processes of the keratocytes became slender and often twisted. Their protoplasm became more electron dense while in some instances cytoplasmic decomposition was also evident (Figs. 8, 9). The nucleus of many keratocytes became pyknotic and occasionally took up a lobular shape (Fig. 9).

A widespread, homogeneous increase of interfibrillar distance was not observed even in severe stromal oedema. Instead, significant increases of interfibrillar distance occurred in patches. These localised regions of stromal oedema were primarily located in the posterior stroma (Figs. 9, 12). A granular material appeared to accumulate between these collagen fibres. The corneas that had worn the contact lens for 2 hours showed minimal stromal oedema with spaces primarily around the keratocytes. The posterior stroma remained normal in these eyes. Invading cells were occasionally encountered in the stroma of the eyes with the longer wearing time. 


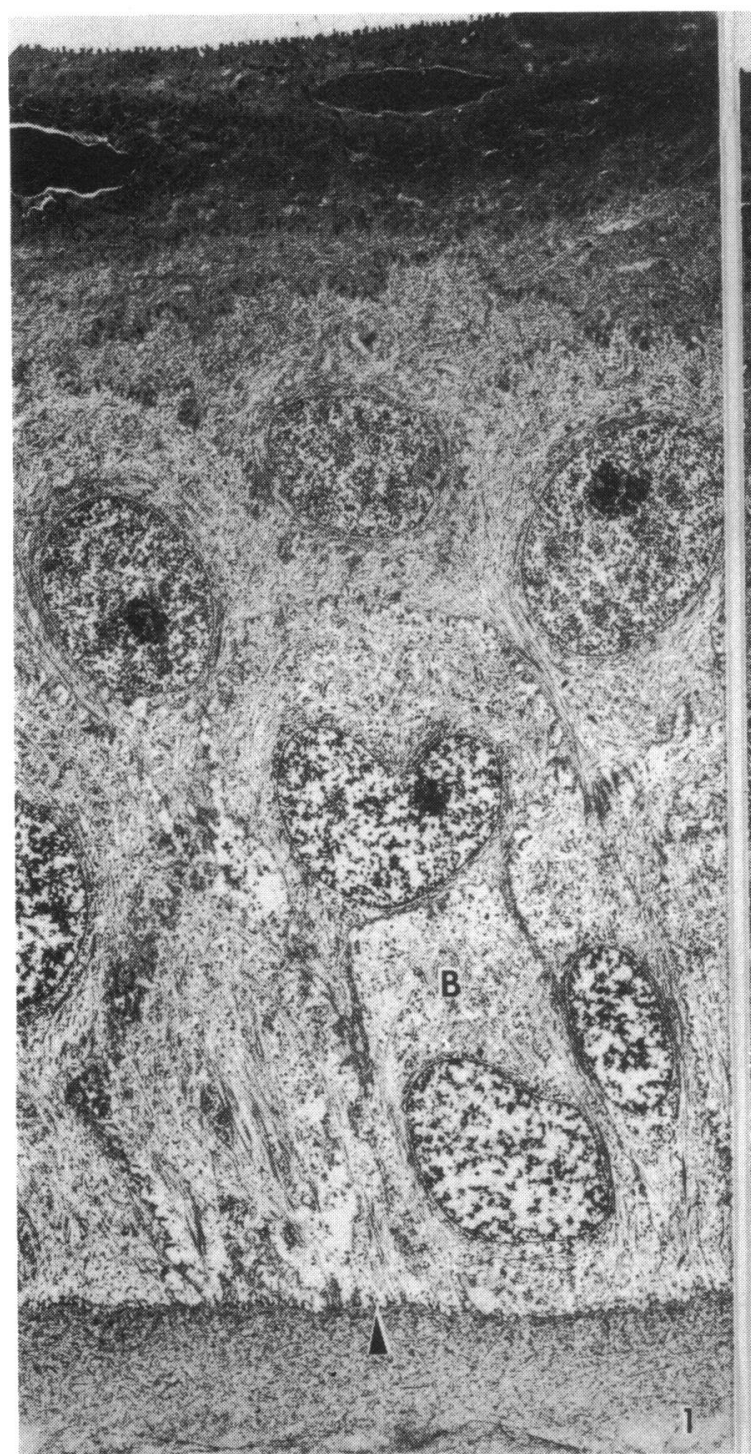

Fig. 1 Normal corneal epithelium. The epithelial cells are tightly packed, with no intercellular spaces. The columnar basal cells $(B)$ are adhering to a basement membrane (triangle). $(\times 3000)$.

POSTERIOR LIMITING LAMINA AND ENDOTHELIUM Rigid contact lens wear of 2 or 24 hours did not induce changes within the posterior limiting lamina (Descemet's membrane) (Fig. 12). This basement membrane maintained its normal thickness and regular texture throughout the width of the cornea (Figs. 11, 12).

While 24 hours of rigid contact lens wear produced a mild endothelial reaction, the shorter 2-hour

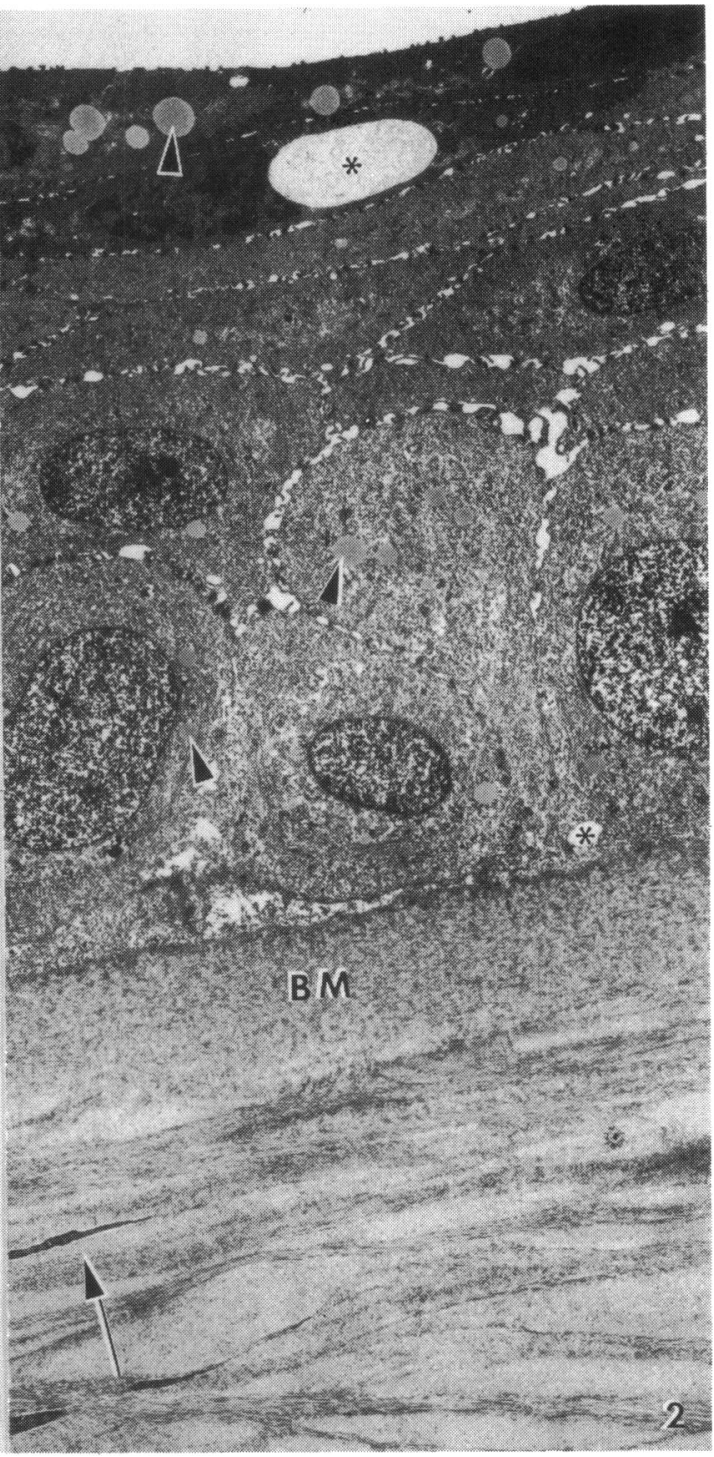

Fig. 2 Mild epithelial oedema. Spaces, many of which contain a granular material, are found between the epithelial cells as well as within (asterisk) these cells. Lipid-like deposits (triangle) are evident in most cells. The epithelial cells have lost some of their normal shape, showing a tendency to flatten. In comparison with the normal epithelial surface the mildly oedematous corneal surface has fewer microvilli.

Except for a slight widening of lamellae around a keratocyte (arrowhead), the anterior stroma with its complex undulating lamellar organisation shows no signs of oedema. Two hours' wear $(\times 3000)$.

wearing time caused no observable endothelial changes. The prolonged wearing time appeared to loosen the adherence of the endothelium to its 
Fig. 3 Detail of oedematous epithelium. Along the surface is a degenerated cell (D). A granular material is found in spaces between the epithelial cells, which project elongated cytoplasmic extensions across these gaps (triangle).

Microvilli have appeared along the cell surfaces (arrowhead).

Twenty-four hours' wear. $(\times 7273)$.

Fig. 4 Detail of advanced oedema. Increased epithelial oedema causes large vesicles $(V)$ to appear. The continued loss of normal cell junctions together with an increased number of intraepithelial microvilli is evident (triangle). Twenty-four hours' wear. $(\times 4364)$.

Fig. 5 Detail of advanced oedema. Apart from spaces within and between epithelial cells, other degenerative signs are lipid-like deposits (triangle) and lysosome-like bodies (L). Twenty-four hours' wear. ( $\times 4364)$.

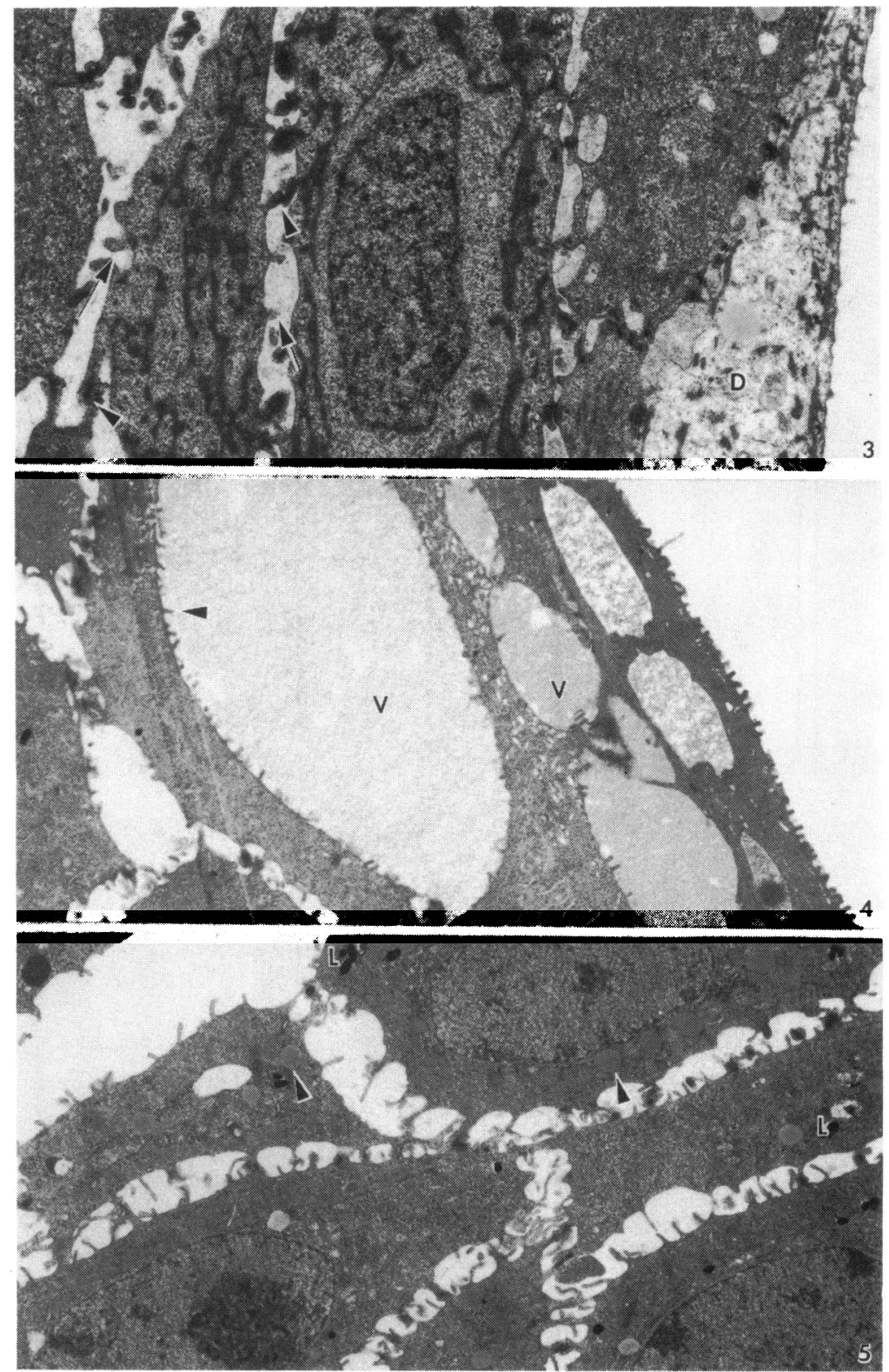

\section{Discussion}

Excellent detailed descriptions of the normal anatomy of the cornea are available, ${ }^{910}$ and the observations made on the control eyes in the present study were in agreement with these earlier reports.

After the contact lenses had been worn for varying times, spaces were seen between but also within the epithelial cells. These spaces contained a granular 

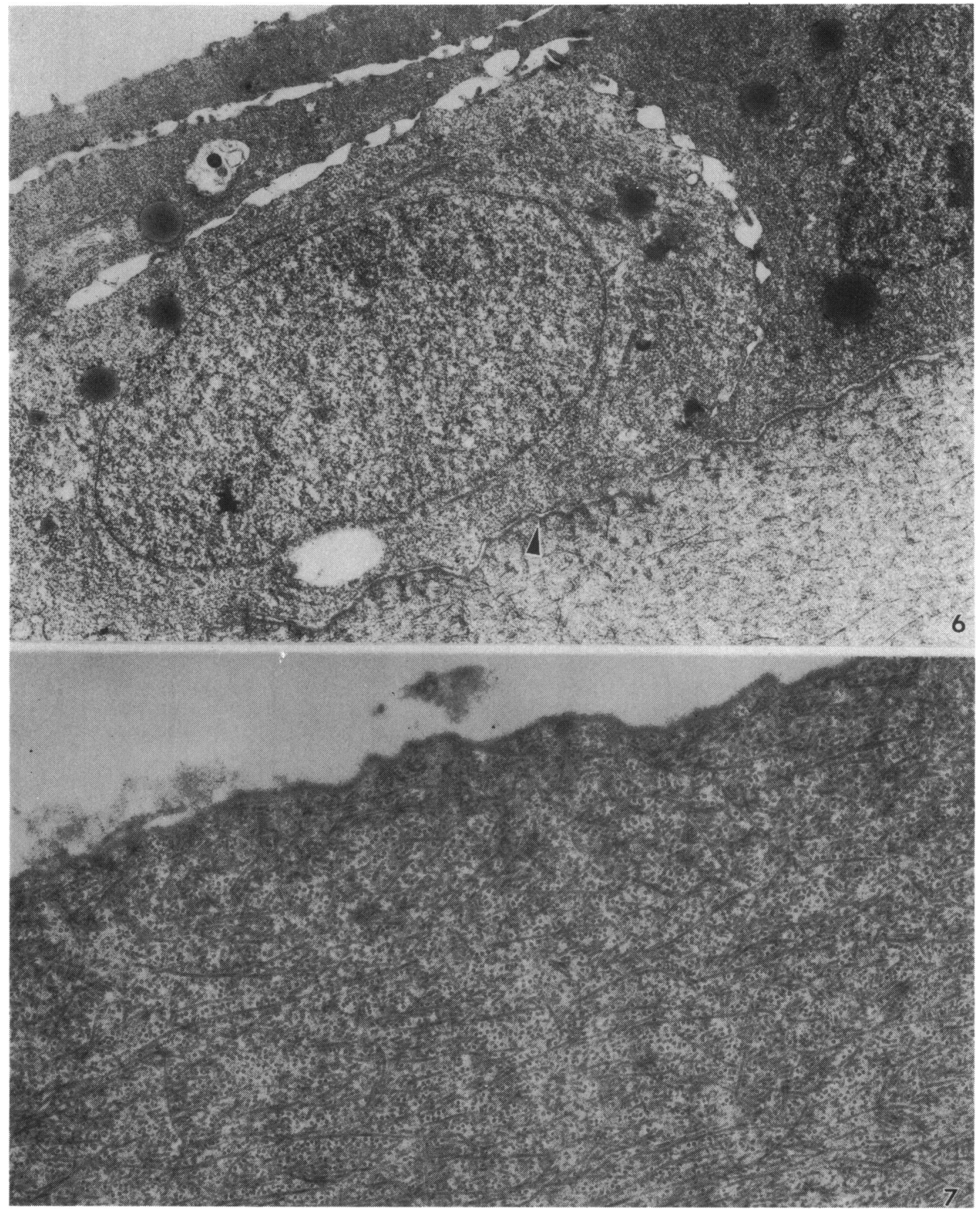

Fig. 6 Epithelial thinning. Premature sloughing of surface cells has reduced the epithelial thickness at this point to 3 layers of degenerating and relatively flat cells. The wavy outline of the basement membrane (triangle) suggests that it is under some stress. Twenty-four hours' wear. $(\times 7000)$.

Fig. 7 Area of denuded cornea. The contact lens had denuded the cornea of epithelial cells in some regions while leaving the intact basement membrane to form the corneal surface. In this illustration as well as Fig. 6 the anterior limiting lamina shows no apparent signs of swelling. Twenty-four hours' wear. $(\times 18000)$. 
Fig. 8 Keratocyte with induced changes in mid to anterior stroma. Although the surrounding stroma appears normal, a keratocyte (C) shows cytoplasmic decomposition. Twenty-four hours' wear. $(\times 3636)$.

Fig. 9 Keratocyte with induced changes in posterior stroma. The nucleus of the keratocyte is pyknotic and the cytoplasm is granular and condensed. Fluid has accumulated around the keratocyte, while it is also evident within the lamellae between collagen fibres (triangles). Twenty-four hours' wear. ( $\times 5818)$.

Fig. 10 Oedema in midstromal region. The stromal swelling has produced a wide separation (S) between 2 adjacent and partially overlapping keratocytes.

Twenty-four hours' wear. ( ×4364).
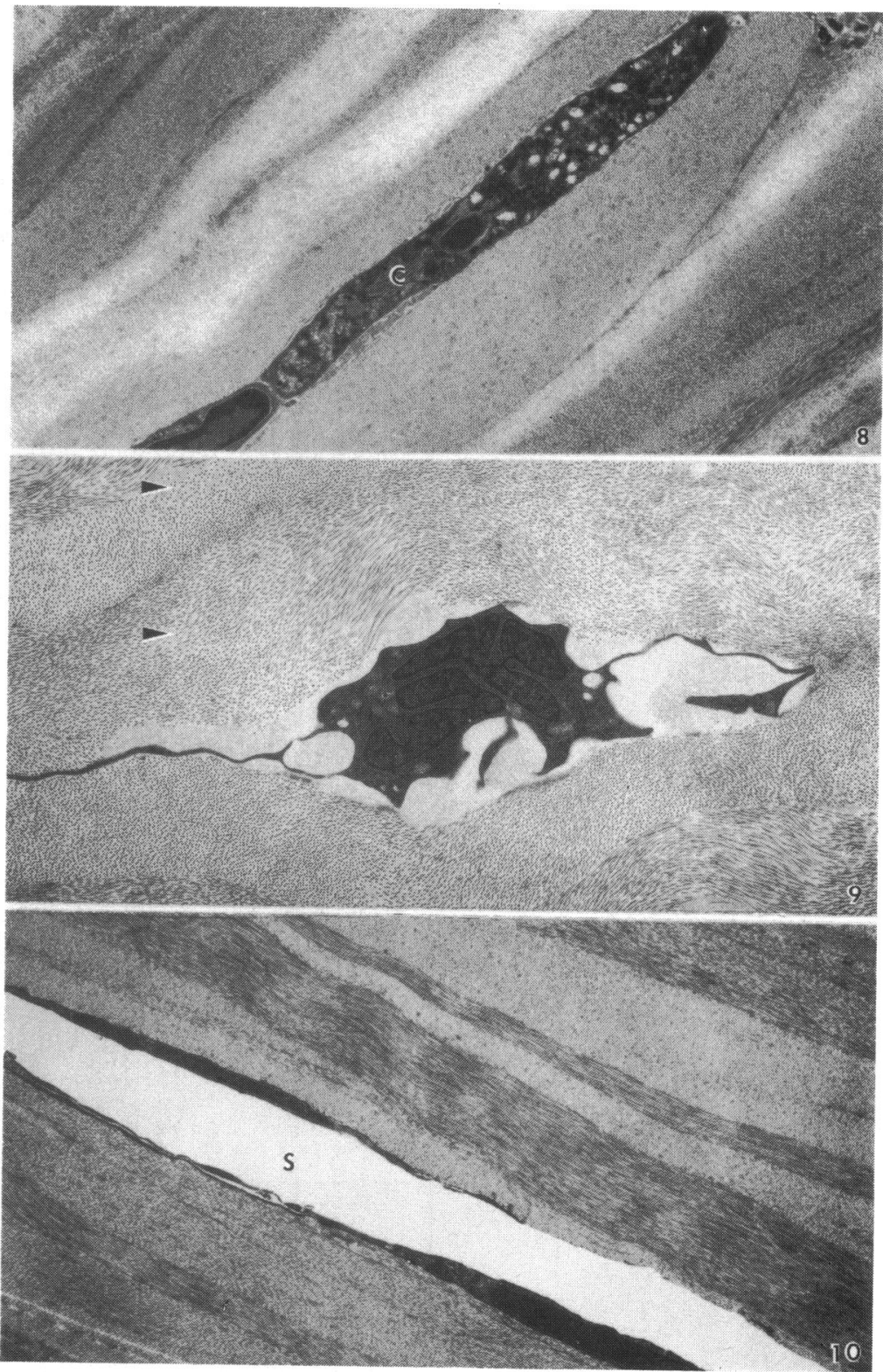

material that is assumed to represent the accumulated fluid in the oedematous epithelium. Epithelial oedema was present after as little as 2 hours of PMMA contact lens wear, but these corneas had little or no stromal oedema. It follows that in epithelial oedema provoked by contact lenses fluid would-at least initially-enter this corneal layer from the tears rather than the stroma. Swelling of the epithelium in a posterior to anterior direction is, however, mostly the case in pathologically induced oedema, which has its cause often in interference of the normal endothelial function. ${ }^{23}$ The early epithelial response may, apart from anxoia, also be due to increased tearing provoked by irritation from contact lenses. Schoessler and Lowther ${ }^{11}$ suggested that epithelial oedema is caused by excessive or hypotonic tears rather than anoxia.

It was significant that after 2 hours of rigid contact 


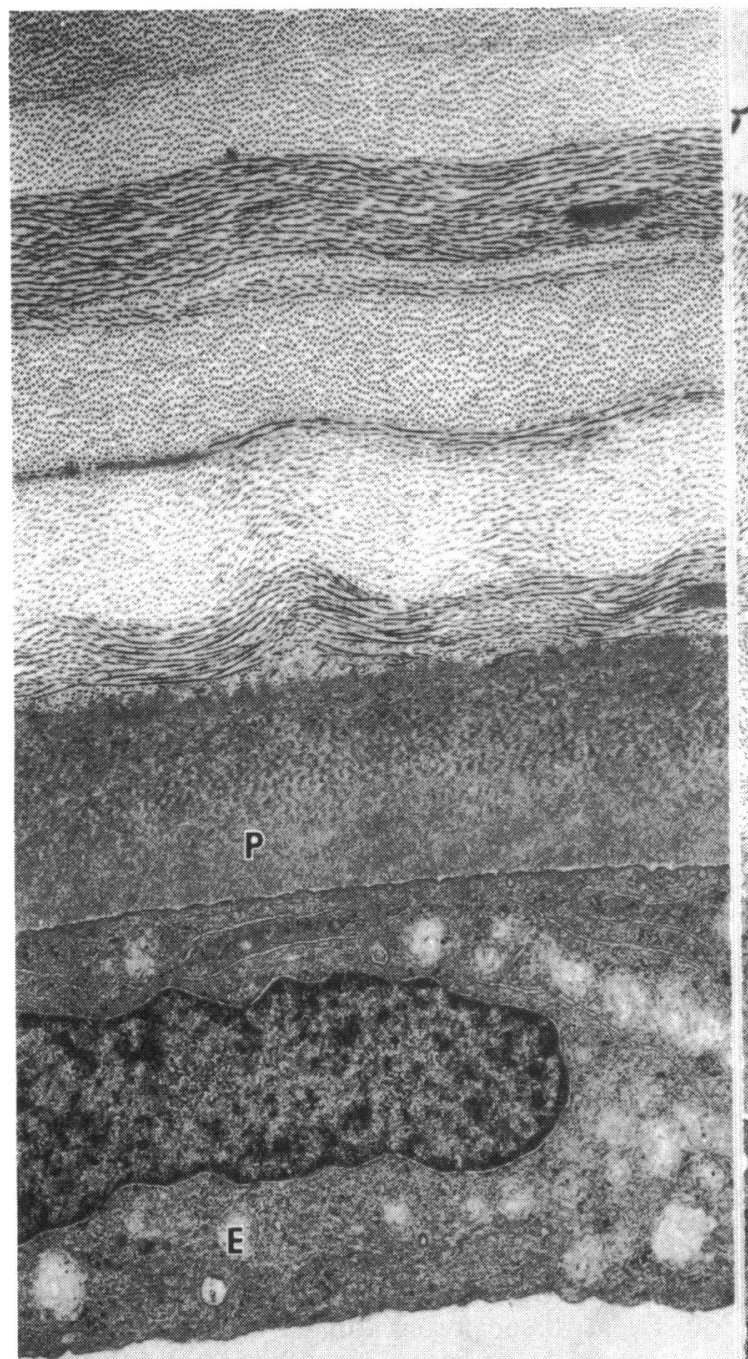

11

Fig. 11 Normal posterior cornea. The endothelial cell (E) lines the posterior limiting lamina $(P)$. The posterior stroma shows a regular uncomplicated lamellar pattern. $(\times 7000)$.

lens wear there were clear signs of adverse cellular reactions. Apart from moderate accumulation of intracellular fluid lipid-like bodies were found within the cells of all epithelial layers. More extended contact lens wear produced an apparent lysosomal activity. These changes suggest that the corneal epithelium is under considerable stress even at an

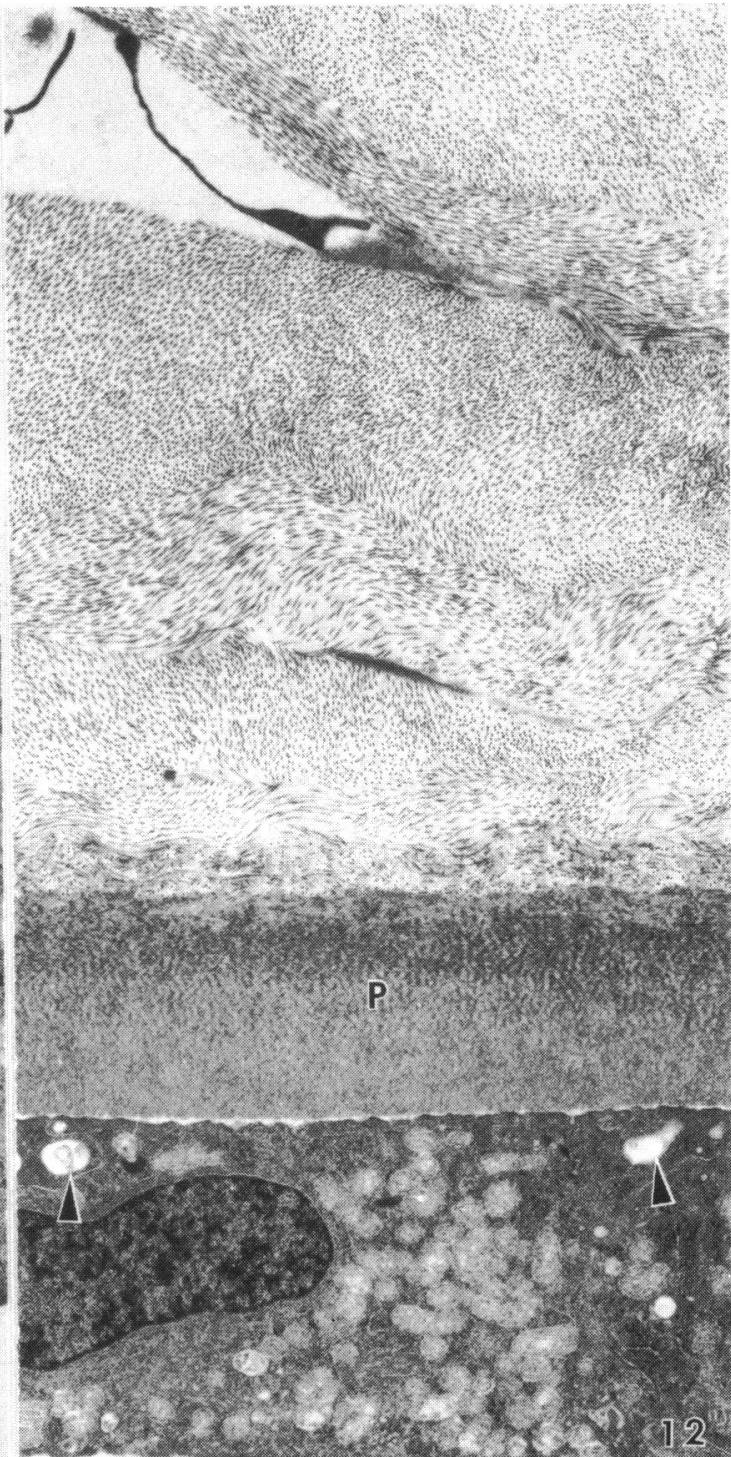

Fig. 12 Oedematous posterior stroma. The posterior stroma exhibits significant oedema, while the posterior limiting lamina $(P)$ appears normal. Endothelial reaction is limited to small pockets of fluid (triangles) within the cells. A slight tendency to endothelial separation from the posterior limiting lamina is evident. Twenty-four hours' wear. $(\times 6000)$

early stage of contact lens wear. The epithelial stress is also expressed by the change in shape of cells. This change appeared to be at least in part produced by fluid entering the epithelium and causing a stretching of the cytoplasm in the area of desmosomal contact with neighbouring cells. This created finger-like projections held together by desmosomes and 
extending across the space between the cells. As the oedema progresses, an increasing number of desmosomes disappeared. Pedler ${ }^{12}$ showed that the surface microvilli are remnant desmosomal contacts, and presumably the microvilli observed within the oedematous epithelium were also caused by a rupture of desmosomal contacts. Goldman and Kuwabara ${ }^{2}$ and Dohlman ${ }^{3}$ reported that the desmosomes appeared unaltered in acute epithelial oedema such as is caused by trauma. The observations made in the present study support this only to the point that the still-present desmosomes were of normal appearance. However, the number of desmosomes had decreased at the same time as microvillus-like structures appeared. This finding appears to support Pedler's theory of the origin of corneal microvilli.

Anoxia has been reported to produce little or no epithelial swelling, ${ }^{45}$ but this fact does not permit us to assume epithelial oedema cannot be provoked by oxygen deprivation. O'Leary et al..$^{5}$ indeed found that patients with anoxic corneas experienced haloes round lights, suggesting that intercellular spaces had occurred due to hypoxia. It is here suggested that the fluid uptake that occurs with oedema is compensated for by a shrinking and flattening of the cells in the corneal epithelium. This theory may further explain the study by O'Leary et al. ${ }^{5}$ who found up to a $20 \%$ decrease in epithelial thickness during recovery from anoxia. It may indicate that during recovery interepithelial fluid is removed faster than the cells return to normal size and shape or that the fluid cell complex occupies less space than the normal healthy cell, and hence an overall shrinkage of the epithelium would occur.

Denuding the cornea of its epithelium by scraping will leave the basement membrane of the epithelium intact. ${ }^{13}$ The present study showed the PMMA lens to be capable to doing the same. In an earlier report the present authors described the histological appearance of contact-lens-induced epithelial injury and stressed the excellent durability of the epithelial basement membrane. ${ }^{6}$

After 2 hours of contact lens wear the stroma showed minimal oedema, while after 24 hours of contact lens wear, which may be equated with a severe case of overwear, the stroma of the cornea showed significant oedema. However, the anterior limiting lamina did not display a detectable increase in interfibrillar distance in any cornea. This observation was in agreement with that of Goldman and Kuwabara, ${ }^{2}$ who reported that the anterior limiting lamina maintained its integrity in swollen corneas. Stromal swelling was therefore unlikely to be caused by leakage of water from the epithelial side of the cornea. Since stromal oedema appeared most significant posteriorly, it appears that it was the result of altered endothelial function rather than a leakage of fluid into the stroma from the epithelium. The earliest sign of stromal oedema in contact lens wearers is frequently the vertical folds or striae, which are caused by oxygen deprivation. ${ }^{8}$ From the results of the present study it is postulated that stromal oedema in the anoxic cornea begins posteriorly, advances in an anterior direction, and is due to endothelial stress. This would explain the early appearance of vertical striae, which are found primarily in the posterior stroma.

The detailed appearance of stromal oedema with increased interfibrillar separation, that is, wide separations (or 'lakes') primarily between lamellae and ample spaces around keratocytes, was essentially in agreement with earlier reports on pathologically swollen corneas. ${ }^{22}$ The significant degenerative changes and possible cell death of keratocytes observed in the present study in the severely oedematous cornea suggest that abuse or overwear of rigid contact lenses may lead to permanent stromal changes. Indeed, the induced changes among keratocytes bore a close resemblance to the changes described in keratocytes in pre-Descemet's dystrophy. ${ }^{14}$

Only prolonged contact lens wear produced a visible endothelial reaction. The changes appeared benign, with primarily small amounts of fluid within the cells but no breakdown of organelles. They were probably reversible. Since zonula occludentes were intact in the oedematous cornea, it may be assumed that little or no fluid entered the cornea between the cells. Perhaps the most adverse endothelial reaction was its apparent reduced adherence to the normal posterior limiting lamina. This loss of adherence may have been a preparation artefact, though the normal appearing organelles within the cell indicated satisfactory tissue preservation.

We acknowledge the assistance of Professor Jack Crawford, Department of Ophthalmology, University of Texas Medical School. He generously provided the corneas used in the present study. We are also grateful to Professor Donald G. Pitts, University of Houston College of Optometry, who offered constructive criticism of the manuscript. Mr Tommy C. Yee gave valuable technical assistance.

\section{References}

1 Kanai A, Kaufman HE. Electron microscopic studies of swollen corneal stroma. Ann Ophthalmol 1973; 5: 178-90.

2 Goldman JN, Kuwabara T. Histopathology of corneal edema. In: Dohlman CH, ed. Corneal Edema. Boston, Little Brown, 1968: 561-79.

3 Dohlman $\mathrm{CH}$. The function of the corneal epithelium in health and disease. Invest Ophthalmol Visual Sci 1971; 10: 383-407.

4 Wilson GS, Fatt I, Freeman RD. Thickness changes in the strom:I of an excised cornea during anoxia. Exp Eye Res 1973; 17 165-71.

5 O'Leary DJ, Wilson G, Henson DB. The effect of anoxia on the 
human corneal epithelium. Am J Optom Physiol Opt 1981; 58: 472-6.

6 Bergmanson JPG, Chu L W-F. (1982) Contact lens induced corneal epithelial injury. Am J Optom Physiol Opt in press.

7 Ruben M. Complications of soft lens wear. In: Ruben M, ed. Sof Contact Lenses. Clinical and Applied Technology. New York: Wiley, 1978: 293-7.

8 Polse KA, Mandell RB. Etiology of corneal striae accompanying hydrogel lens wear. Invest Ophthalmol Visual Sci 1976; 15: 553-6.

9 Hogan MJ, Alvarado JA, Wedell JE. Histology of the Human Eye. Philadelphia, London, Toronto: Saunders, 1971; 55-111.
10 Kuwabara T. Current concepts in anatomy and histology of the cornea. Contact and Intraocular Lens Medical Journal 1978; 4: 101-32.

11 Schoessler JP, Lowther GE. Slit lamp observations of corneal edema. Am J Optom Physiol Opt 1971; 48: 666-71.

12 Pedler C. The fine structure of the corneal epithelium. Exp Eye Res 1962; 1: 286-9.

13 Hirst LW, Kenyon KR, Fogle J, Stark WJ. Corneal epithelial adhesion studies. Aust J Ophthalmol 1981; 9: 103-11.

14 Curran R, Kenyon K, Green R. Pre-Descemet's membrane corneal dystrophy. Am J Ophthalmol 1974; 77: 711-6. 